Check for updates

Cite this: J. Mater. Chem. C, 2021, 9, 1755

Received 22nd July 2020,

Accepted 30th September 2020

DOI: $10.1039 / \mathrm{d} 0 \mathrm{tc0} 03477 \mathrm{k}$

rsc.li/materials-c

\title{
Bulk-heterojunction photocapacitors with high open-circuit voltage for low light intensity photostimulation of neurons $\dagger$
}

\author{
Shashi Bhushan Srivastava, (D) $\ddagger^{a}$ Rustamzhon Melikov, (D) $\ddagger^{a}$ Erdost Yildiz, (D) ${ }^{b}$ \\ Ugur Meric Dikbas, ${ }^{c}$ Sadra Sadeghi, (D) d Ibrahim Halil Kavakli, (D) ${ }^{c e}$ Afsun Sahin (D) bf \\ and Sedat Nizamoglu iD *ag
}

\begin{abstract}
High-level transduction control of light to bioelectricity is an important goal for the realization of superior neuron-device interfaces that can be used for regulating fundamental cellular processes to cure neurological disorders. In this study, a single-junction, wireless, and capacitive-charge-injecting optoelectronic biointerface with negligible faradaic reactions by using a high open-circuit voltage $(0.75 \mathrm{~V})$ bulk heterojunction of PTB7Th:PC71BM is designed and demonstrated. The biointerface generates a 2-fold higher photocurrent in comparison with P3HT:PC61BM having an open-circuit voltage of $0.55 \mathrm{~V}$. Furthermore, we observed that light intensity is logarithmically correlated with the open-circuit voltage of solar cells, and the photovoltage of the biointerfaces varies the switching speed of capacitive charge-transfer. Finally, pulse trains of capacitive stimuli at a low light intensity of $20 \mathrm{~mW} \mathrm{~cm}{ }^{-2}$ elicit action potential generation in primary hippocampal neurons extracted from E15-E17 Wistar Albino rats. These findings show the great promise of high open-circuit voltage bulk heterojunction biointerfaces for non-genetic, all-optical and safe modulation of neurons.
\end{abstract}

\section{Introduction}

Light-induced stimulation of neurons has high potential to understand the complex communication in neural networks, regulate the physiological activities and restore lost functions. ${ }^{1-7}$ Organic photovoltaic biointerfaces offer a configurable solution for light-induced stimulation of neurons. ${ }^{8}$ Advantageously, they convert light to bioelectrical stimuli without any need for electrical power supply and integrated complex electronics.

\footnotetext{
${ }^{a}$ Department of Electrical and Electronics Engineering, Koc University, Istanbul 34450, Turkey. E-mail: snizamoglu@ku.edu.tr

${ }^{b}$ Koç University Research Center for Translational Medicine, Koc University, Istanbul 34450, Turkey

${ }^{c}$ Molecular Biology and Genetics, College of Science, Koc University, Istanbul 34450, Turkey

${ }^{d}$ Graduate School of Materials Science and Engineering, Koc University, Istanbul 34450, Turkey

${ }^{e}$ College of Engineering, Chemical and Biological Engineering, Koç University, Istanbul 34450, Turkey

${ }^{f}$ Department of Ophthalmology, Koç University Medical School, Koç University, Istanbul 34450, Turkey

${ }^{g}$ Graduate School of Biomedical Sciences and Engineering, Koc University, Istanbul 34450, Turkey

$\dagger$ Electronic supplementary information (ESI) available. See DOI: 10.1039/ d0tc03477k

\# S. B. S. and R. M. contributed equally to this work.
}

Moreover, proper band engineering can facilitate control of the faradaic and capacitive charge transfer mechanisms, among which capacitive mechanisms are more preferred due to the perturbation via electromagnetic charge-ion interactions without any chemical reaction. ${ }^{9}$

One of the most important figures of merits affecting the optoelectronic properties of the photovoltaic substrates and simultaneously the photostimulation properties is the opencircuit voltage. For the photostimulation of cells, photodiodes connected in series and tandem architecture were utilized to change the open-circuit voltage $\left(V_{\text {oc }}\right)^{6,10}$ Alternatively, bulk heterojunctions can overcome the limitation of charge carrier diffusion in organic semiconductors and enable significant enhancement in the generation of photo-induced charges that can be effectively used for the stimulation of neurons. ${ }^{11}$ Moreover, it can also allow for the control of optoelectronic and bioelectronic properties of biointerfaces by varying the photoactive layer. So far, P3HT:PCBM, P3OT:N2200, PDPP3T:PCBM, and P3HT:N2200 bulk heterojunctions were independently examined for photostimulation of cells. ${ }^{10,12-16}$

In this study, we explore a high open-circuit voltage $\left(V_{\mathrm{oc}}\right)$ photovoltaic material consisting of a donor copolymer PTB7-Th (poly[4,8-bis(5-(2-ethylhexyl)thiophen-2-yl)benzo[1,2- $\left.b ; 4,5-b^{\prime}\right]$ dithiophene-2,6-diyl-alt-(4-(2-ethylhexyl)-3-fluorothieno[3,4- $b]$ thiophene-)-2-carboxylate-2-6-diyl)] and an acceptor small molecule 
PC71BM ([6,6]-phenyl C71 butyric acid methyl ester) in a singlejunction biointerface design for the first time, while addressing the important aspect of safe neural photostimulation via capacitive charge transfer. Remarkably, we demonstrate that light-intensity can control the switching speed of capacitive currents in the wireless and free-standing mode configuration, in addition to the stimuli strength. Moreover, the higher open-circuit voltage biointerface advantageously facilitates faster switching of capacitive currents in comparison with the lower- $V_{\text {oc }}$ (P3HT:PCBM) biointerface. It also facilitates the photostimulation of neurons at low-light intensity levels. Therefore, electronic and optical tuning of open-circuit voltage enables for the control of switching speed and strength of neuro-modulating capacitive stimuli.

\section{Experimental}

\section{Biointerface fabrication}

The two types of biointerfaces were fabricated by changing the photoactive layer with different $V_{\text {oc }}$ values keeping the indium tin oxide (ITO)/zinc oxide ( $\mathrm{ZnO}$ ) electrode the same. The low- $V_{\text {oc }}$ photoactive layer consists of a blend of $95.7 \%$ regioregular P3HT (poly(3-hexylthiophene-2,5-diyl)) and >99\% pure PC61BM ([6,6]-phenyl-C61-butyric acid methyl ester). The high- $V_{\text {oc }}$ photoactive layer was prepared by blending a donor copolymer poly[4,8-bis(5-(2-ethylhexyl)thiophen-2-yl)benzo[1,2$b ; 4,5$ - $\left.b^{\prime}\right]$ dithiophene-2,6-diyl-alt-(4-(2-ethylhexyl)-3-fluorothieno $[3,4-b]$ thiophene-)-2-carboxylate-2-6-diyl] (PTB7-Th) and an acceptor molecule [6,6]-phenyl-C71-butyric acid methyl ester (PC71BM). The ITO coated glass substrates, P3HT, PTB7-Th, PC61BM, and PC71BM were purchased from Ossila Ltd. Zinc acetate dihydrate, ethanolamine, 2-methxyethanol, chlorobenzene, 1,2-dichlorobenzene, and molybdenum trioxide were purchased from Sigma Aldrich. All the materials and chemicals were used as received.

To fabricate the biointerfaces, the ITO coated glass substrates were sequentially cleaned using $1 \%$ by volume Hellmanex soap solution (Ossila Ltd), de-ionized water, acetone, and isopropanol using ultrasonication. The cleaned substrates were dried in oven at $100{ }^{\circ} \mathrm{C}$ and treated with UV-ozone for $15 \mathrm{~min}$ before the coating. The pre-cleaned substrates were spin coated at $2000 \mathrm{rpm}$ for $60 \mathrm{~s}$ with $\mathrm{ZnO}$ precursor sol-gel solution. The coated substrates were annealed at $250{ }^{\circ} \mathrm{C}$ for $15 \mathrm{~min}$ to get $50 \mathrm{~nm}$ of $\mathrm{ZnO}$ thin film. The reference photoelectrode (BI-1) was completed by spin coating of P3HT:PC61BM (1:1 weight ratio) solution in $o$-dichlorobenzene on the ITO/ZnO substrate at $1000 \mathrm{rpm}$ for $90 \mathrm{~s}$ and annealed at $150{ }^{\circ} \mathrm{C}$ for $15 \mathrm{~min}$. However, the high- $V_{\mathrm{oc}}$ photoelectrode (BI-2) was prepared by spin coating of PTB7-Th : PC71BM (1:1.5 weight ratio) solution in chlorobenzene on ITO/ZnO substrate at $600 \mathrm{rpm}$ for $120 \mathrm{~s}$ and then dried under nitrogen for 2 h. ${ }^{17}$ The thickness of the various layers was confirmed using a SEM cross-section image.

\section{Optical and microscopic characterization}

The optical absorbance for the BI-1 and BI-2 biointerfaces is determined using a UV-Vis spectrophotometer. The microscopic images of the photoelectrode surfaces are investigated using an atomic force microscope (AFM). Scanning electron microscopy is used to take cross-sectional images for the optimization of the various layer thicknesses.

\section{Electrochemical measurement}

To study the interfacial charge transfer processes, impedance spectroscopy (IS) measurements are carried on biointerfaces with different photoactive layers interfaced with electrolyte medium under light conditions. This involves measuring the electrical impedance and phase angle obtained with sinusoidal voltage excitation of the electrode. The measurement is made over a broad frequency range, between 1 and $10^{5} \mathrm{~Hz}$, and the samples are probed under $10 \mathrm{mV}\left(V_{\mathrm{rms}}\right)$ AC perturbation at zero dc bias under light conditions (at a wavelength of $445 \mathrm{~nm}$ with an intensity level of $10 \mathrm{~mW} \mathrm{~cm}^{-2}$ ). The magnitude of the AC perturbation is kept sufficiently small so that a linear current-voltage response is obtained at each frequency. ${ }^{18}$ Using IS, multiple charge carrier accumulation and charge transport can be distinguished at various interfaces in the device according to their response against an externally applied AC signal. For quantitative analysis, the impedance data are modeled using an equivalent electrical circuit with the circuit elements, which are assigned to various physical phenomena in the device. ${ }^{9,19-21}$ The fitted parameters allow us to calculate the capacitance between the photoelectrode and electrolyte (extracellular medium) interface. ${ }^{22}$ In order to measure photocurrent and photovoltage, we utilized the 2-probe configuration of electrochemical measurement under short-circuit conditions keeping $0 \mathrm{~V}$ bias between the working and counter electrode. The photoactive area of $1 \mathrm{~cm}^{2}$ was fixed by using a Redoxme Spectro-EFC cell set-up.

\section{Cytotoxicity assessment}

Biocompatibility of the photoelectrodes was assessed by measuring the mitochondrial activity of SH-SY5Y cells using MTT assay. The photoelectrodes and ITO control are sterilized using $70 \%$ ethanol and $30 \mathrm{~min}$ UV-C exposure. In a 6-well plate, $0.3 \times 10^{6}$ SHSY-5Y cells were seeded onto samples and incubated at $37{ }^{\circ} \mathrm{C}$ and $5 \% \mathrm{CO}_{2}$. Cytotoxic control samples are prepared with ITO samples that were incubated with growth medium containing $10 \%$ dimethyl sulfoxide (DMSO). After 48 hours, the growth medium is discarded and $1 \mathrm{mg} \mathrm{ml}$ 3-(4,5-dimethylthiazol-2-yl)-2,5-diphenyltetrazolium bromide (MTT) is added in serum-free DMEM. Samples were incubated with MTT solution for $4 \mathrm{~h}$ to allow formazan formation. Finally, the MTT solution is removed and formazan salts are dissolved using EtOH/DMSO. The amounts of formazan are measured using a microplate reader (BioTek, Synergy H1) in a 96-well plate. Relative absorbance of the photoelectrode samples to the ITO control is calculated to determine the relative biocompatibility.

\section{Immunolabeling and fluorescence microscopy}

SH-SY5Y cells were grown on pre-sterilized substrates in a 6-well plate as explained in the 'Cytotoxicity assessment' section. After $48 \mathrm{~h}$ of incubation at $37{ }^{\circ} \mathrm{C}$ in a $5 \% \mathrm{CO}_{2}$ incubator, the growth 
medium was discarded. For cell fixation, 4\% paraformaldehyde was added to phosphate-buffered saline (PBS) solution and the samples were incubated at $37{ }^{\circ} \mathrm{C}$ for $30 \mathrm{~min}$. Fixed cells were rinsed three times with PBSt (PBS containing 0.1\% Triton $\mathrm{X} 100$ ) and then blocked using PBSt containing 5\% bovine serum albumin. Anti-beta III tubulin (ab78078, Abcam) primary antibody was used as neuronal marker labelling the cytoskeleton of SH-SY5Y cells. Samples were incubated with anti-beta III tubulin antibody for $2 \mathrm{~h}$ and washed three times with PBSt. Alexa Fluor conjugated goat anti-mouse IgG H\&L (ab150113, Abcam) was used as a secondary antibody and DAPI stain was used to detect nuclei. After $1 \mathrm{~h}$ of incubation with secondary antibody and DAPI, the samples were washed with PBSt and mounted by Mowiol. Imaging was performed using a fluorescence microscope (ObserverZ1, Zeiss).

\section{Primary hippocampal neuron isolation and culture}

Hippocampal regions were extracted from decapitated E15-E17 Wistar Albino rats and were placed immediately in ice-cold Hank's Balanced Salt Solution (HBSS, Thermo Fisher Scientific, MA, USA). The hippocampi were incubated in $0.25 \%$ Trypsin-EDTA solution (Thermo Fisher Scientific, MA, USA) with 2\% DNase-I supplement (NeoFroxx, Einhausen, Germany) for 20 minutes in a $37{ }^{\circ} \mathrm{C}$ incubator. Then the cells were centrifuged and the supernatant was changed with Dulbecco's Modified Eagle Medium/Nutrient Mixture F-12 (DMEM/F12 Thermo Fisher Scientific, MA, USA) supplemented with $10 \%$ fetal bovine serum (FBS, Heat Inactivated, GE Healthcare, IL, USA) and 1\% penicillin/streptomycin (Thermo Fisher Scientific, MA, USA). DMEM/F12 was removed and Neurobasal Medium (NBM, Thermo Fisher Scientific, MA, USA) supplemented with B27, L-glutamine, $\beta$-mercaptoethanol, and glutamate (Thermo Fisher Scientific, MA, USA) was added to the cell pellet. The cells were triturated and were passed through a $70 \mu \mathrm{m}$ cell strainer. The homogenous cell solution was seeded in poly-Dlysine (PDL, Sigma-Aldrich, MO, USA) coated substrates. After 3 days of incubation of the cells on the substrates in a $37{ }^{\circ} \mathrm{C}$ incubator with $5 \%$ carbon dioxide, the media of the cells on the substrates were changed with NBM supplemented with cytosine arabinoside (Sigma-Aldrich, MO, USA) to inhibit growth of glial cells. After 24 hours incubation with cytosine arabinoside, the media were changed with NBM. Therefore, for maturation of primary hippocampal neural cells, the cells on the substrates were incubated in NBM between 3 and 5 days before patch clamp experiments. ${ }^{23-25}$ Then, the hippocampal neurons were cultures on photoelectrode substrates for future experiments.

\section{Electrophysiology measurement}

The photostimulation was performed on a set-up comprising an Olympus T2 upright microscope placed inside a Faraday cage to prevent electrical noise. An EPC 800 patch clamp amplifier (HEKA Elektronik) was used for the photocurrent measurements. For the light stimulation, we used a blue light emitting diode (LED) (M450LP1, Thorlabs, NJ, USA) with nominal wavelength of $445 \mathrm{~nm}$ (Fig. S1, ESI $\dagger$ ). The LED was driven by a DC2200 - High-Power 1-Channel LED Driver with Pulse
Modulation (Thorlabs, NJ, USA). The FWHM of the blue light spectrum is $16.7 \mathrm{~nm}$ (Fig. S1, ESI $\dagger$ ). A power meter (Newport 843-R) was used to measure the exact power of light reaching at the interface. The illumination was focused on a water immersion objective $(40 \times / 0.8 \mathrm{~W}$, inf/0/FN 26.5) from the ITO (a transparent electrode) side of the photoelectrode. Pulled patch pipettes of 8-12 $\mathrm{M} \Omega$ were used to investigate the whole-neuron cells under giga-ohm seal. Extracellular solution (Artificial Cerebrospinal Fluid, aCSF) is prepared as follows: $140 \mathrm{mM} \mathrm{NaCl}, 3 \mathrm{mM} \mathrm{KCl}, 1 \mathrm{mM} \mathrm{MgCl}_{2}, 2 \mathrm{mM} \mathrm{CaCl}_{2}, 10 \mathrm{mM}$ HEPES, and $10 \mathrm{mM}$ Glucose. Osmolarity was adjusted to 290 mOsm and $\mathrm{pH}$ was adjusted to 7.4 with $\mathrm{NaOH}$. Internal cellular solution (ICS) is prepared as follows: $140 \mathrm{mM} \mathrm{KCl}$, $2 \mathrm{mM} \mathrm{MgCl}_{2}, 10 \mathrm{mM}$ HEPES, $10 \mathrm{mM}$ EGTA, and $2 \mathrm{mM} \mathrm{Mg-ATP.}$ Osmolarity was adjusted to $270 \mathrm{mOsm}$ and $\mathrm{pH}$ was adjusted to 7.3 with $\mathrm{KOH}$. ICS was used to fill the patch pipettes during the measurement. An Olympus T2 upright microscope and a digital camera were used in the electrophysiology set-up to monitor the cells. The stimulations of the primary hippocampal neurons were effective for a couple of hours and after that they lost their excitability because of the damage by the patched microelectrode.

\section{Results and discussion}

\section{Photocapacitor biointerface structures}

Light-induced capacitive charge-transfer is a safe method for neural photostimulation. For capacitive charge-transfer, the photogenerated charge carriers need to be dissociated and directed toward the semiconductor-electrolyte interface to induce a displacement current. For that bulk heterojunction, photovoltaics provide an appropriate approach. We selected P3HT:PC61BM and PTB7-Th:PC71BM as the low- and high- $V_{\text {oc }}$ bulk heterojunction photovoltaic materials, respectively (Fig. 1a). To generate capacitive stimuli by using the photovoltaic materials, the biointerfaces were prepared in the device forms of ITO/ZnO/P3HT:PC61BM and ITO/ZnO/PTB7Th:PC71BM denoted as BI-1 and BI-2, respectively (Fig. 1a).

To measure the open-circuit voltage of the biointerfaces, we prepared the photovoltaic devices in the following configuration: $\mathrm{BI}-1 / \mathrm{MoO}_{x} / \mathrm{Ag}$ and $\mathrm{BI}-2 / \mathrm{MoO}_{x} / \mathrm{Ag}$. We illuminated them under one-sun AM1.5G solar radiation, and the open-circuit voltages correspond to 0.55 and $0.75 \mathrm{~V}$ for $\mathrm{BI}-1$ and $\mathrm{BI}-2$, respectively (Fig. 1b). Ideally, the open-circuit voltage $\left(V_{\mathrm{oc}}\right)$ for the bulk heterojunction organic solar cells is defined as $V_{\mathrm{Oc}}=\left[\left(\mathrm{HOMO}_{\mathrm{D}}-\right.\right.$ $\left.\left.\mathrm{LUMO}_{\mathrm{A}}\right) / e\right]-0.3$ under one-sun AM1.5G solar radiation, where $\mathrm{HOMO}_{\mathrm{D}}$ is defined as the highest occupied molecular orbital (HOMO) of the donor material, $\mathrm{LUMO}_{\mathrm{A}}$ is defined as the lowest unoccupied molecular orbital (LUMO) of the acceptor material, $q$ is the electron charge, and 0.3 is the correction factor. ${ }^{26,27}$ Under the ideal case, the open-circuit voltage corresponds to $0.7 \mathrm{~V}$ and 1.0 V for BI-1 and BI-2, respectively. Because of the factors such as mobility, lifetime, recombination of charge carriers, etc. they deviate from this ideal value within the range of $0.55-0.60 \mathrm{~V}$ and $0.72-0.85 \mathrm{~V}$ for P3HT:PC61BM and PTB7-Th:PC71BM devices, matching with BI-1 and BI-2, respectively. ${ }^{19,28-33}$ 
a
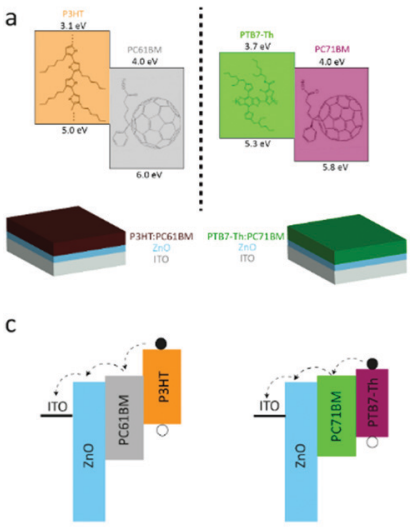

Biointerface-1 (BI-1)

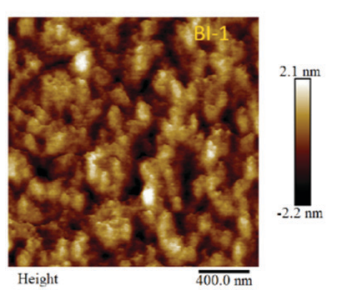

b

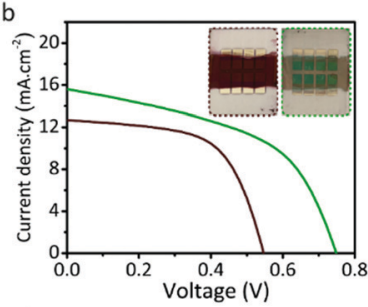

d
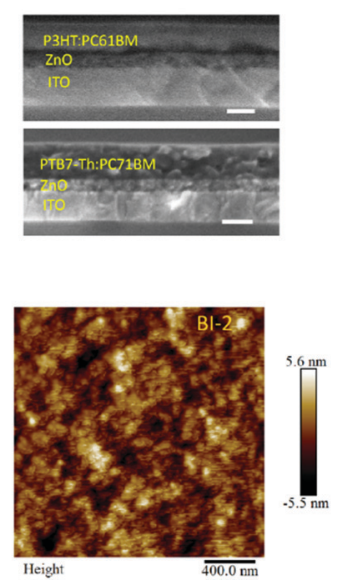

Fig. 1 (a) Energy levels of the bulk heterojunction photovoltaic materials of P3HT:PC61BM and PTB7-Th:PC71BM. Schematic of the device structures of the biointerface 1 denoted as $\mathrm{BI}-1$ (on the left) and biointerface 2 denoted as BI-2 (on the right). (b) Current and voltage characteristics of the solar cell devices. The solar cell devices have the following architectures: $\mathrm{BI}-1 / \mathrm{MoO}_{x} / \mathrm{Ag}$ (dark red) and $\mathrm{BI}-2 / \mathrm{MoO}_{x} / \mathrm{Ag}$ (dark green). Left and right insets show the photographs of the $\mathrm{BI}-1$ and $\mathrm{BI}-2$ based solar cells, respectively. (c) Energy band diagram of $\mathrm{BI}-1$ and $\mathrm{BI}-2$. (d) Cross-sectional SEM image of BI-1 and BI-2. Scale bar: $100 \mathrm{~nm}$ (e) AFM micrograph (surface height profile) of P3HT:PC61BM (BI-1) thin film and (f) PTB7-Th:PC71BM (BI-2) thin film for $2 \times 2 \mu \mathrm{m}^{2}$ scan area.

After exciton dissociation by the bulk heterojunction, the free electron and hole need to be further separated for displacement current generation. For that, the bulk heterojunction composites were interfaced with $\mathrm{ZnO}$ and ITO layers (Fig. 1a). The energy band diagrams of the structures in Fig. 1c showed that the photogenerated charge carriers can be effectively separated for capacitive charge transfer. For a proper comparison of bulk heterojunction performances, the thickness of the BI-1 and BI-2 was kept at similar levels. According to the cross-sectional scanning electron microscopy (SEM) measurements, the thickness of BI-1 and BI-2 corresponds to $\sim 115 \mathrm{~nm}$ and $\sim 119 \mathrm{~nm}$, respectively, and the $\mathrm{ZnO}$ layer thickness was $\sim 50 \mathrm{~nm}$ (Fig. 1d). The surface morphologies of the thin films were characterized by using atomic force microscopy (Fig. 1e and f), and we observed that the morphology of the BI-2 thin film exhibits smaller intermixed domains of donor and acceptor molecules with better homogeneity, which is beneficial for effective charge separation.

\section{Baseline parameters of biointerfaces}

The baseline parameters of the photocapacitor showing the ability of the effective photostimulation are the quantification of photocurrent and charge per unit area. Hence, we measured the electrochemical photoresponse of the $\mathrm{BI}-1$ and $\mathrm{BI}-2$ biointerfaces under the two-probe configuration that allows understanding the actual potential of the photoelectrode in terms of photocurrent and photovoltage. In this configuration (Fig. 2a), an ITO electrode is used as the working electrode, and a platinum electrode that is shorted with $\mathrm{Ag} / \mathrm{AgCl}$ is used as the counter electrode $\left(\sim 7 \mathrm{~mm}^{2}\right.$ area). During the measurement, no voltage was applied to the working electrode and current was monitored over time under light-pulses of $10 \mathrm{~ms}$ with $50 \mathrm{~mW} \mathrm{~cm}{ }^{-2}$ intensity illuminated on the photoactive area of $1 \mathrm{~cm}^{2}$, which was exposed to the aCSF electrolyte. For comparison between the composites, it is preferred to have similar photon absorption by the biointerfaces. Fig. $2 \mathrm{~b}$ indicates the absolute absorption spectra of BI-1 and BI-2, and we selected the wavelength of $445 \mathrm{~nm}$ for optical excitation of the biointerfaces, at which both substrates have similar absorbance levels.

Fig. 2c shows the photocurrent per unit area of the biointerfaces (BI-1 and BI-2) measured between the working electrode (ITO) and counter electrode $(\mathrm{Pt})$ using pulsed $(10 \mathrm{~ms}) 50 \mathrm{~mW} \mathrm{~cm} \mathrm{~cm}^{-2}$ intensity illumination of blue light. The observable two-spikes in the opposite directions matching with the turn-on and -off times point out a capacitive photocurrent. The peak photocurrent density was observed to be 168 and $511 \mu \mathrm{A} \mathrm{cm}{ }^{-2}$ for BI-1 and BI-2 biointerfaces, respectively. The high- $V_{\mathrm{oc}}$ biointerface shows almost a 3-times increase in the photocurrent density compared to the low- $V_{\text {oc }}$ biointerface. The corresponding charge densities were found to be $0.32 \mu \mathrm{C} \mathrm{cm}^{-2}$ and $0.46 \mu \mathrm{C} \mathrm{cm}^{-2}$ for BI-1 and BI-2 biointerfaces. The increase in the charge density is about 1.4 times, which is not at the same level with the increase of photocurrent density. This can be attributed to the faster switching speed of the BI-2 than BI-1 biointerface with respect (a)

(c)

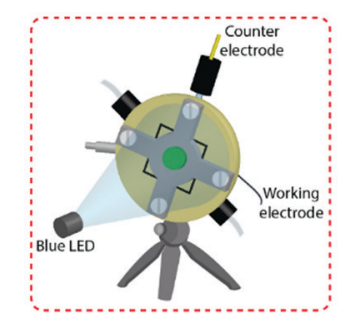

c)

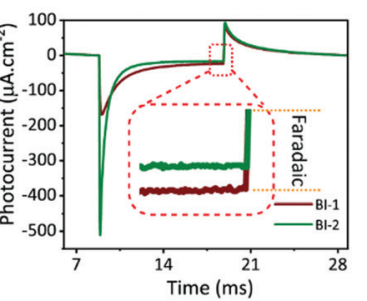

(b)

(d)

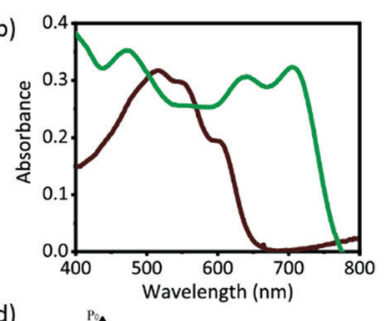

Fig. 2 (a) Schematic illustrating the electrochemical measurement configuration of the photoelectrode in an aCSF solution. A blue LED (pulsed) is used to illuminate and activate the biointerface. (b) Absorbance spectra of $\mathrm{BI}-1$ and $\mathrm{BI}-2$. (c) Photocurrent density comparison of $\mathrm{BI}-1$ and $\mathrm{BI}-2$ biointerfaces under blue light $\left(10 \mathrm{~ms}\right.$ pulse, $445 \mathrm{~nm}, 50 \mathrm{~mW} \mathrm{~cm} \mathrm{~cm}^{-2}$ light intensity). The inset shows the faradaic current components of biointerfaces. The dark red color represents $\mathrm{BI}-1$ and the dark green color represents $\mathrm{BI}-2$ biointerface. (d) Schematic showing the movement of the photogenerated charge carriers in the biointerfaces. 
to the pulsed illumination. Besides, a low level of direct electron or hole transfer via faradaic reactions was observed for BI-1 and BI-2 (Fig. 2c inset). The BI-1 biointerface shows a $24 \mu \mathrm{A} \mathrm{cm}{ }^{-2}$ faradaic current corresponding to almost $14 \%$ of the peak photocurrent and $\mathrm{BI}-2$ has a negligible faradaic component less than $3 \%$ of its peak photocurrent. Since the hole-capturing P3HT and PTB7-Th molecules had energy levels above the water windows and electrons are mainly accumulated inside the device structure, the faradaic charge-transfer processes were limited.

The dissociation of the photogenerated excitons leads to both open-circuit voltage inside the photovoltaic device and interfacial photovoltage between the biointerface and aCSF. To quantitatively analyze the switching properties of the biointerface, we defined the rise and fall times as the time of electrode/ electrolyte photovoltage $\left(V_{\mathrm{ph}}\right)$ reaching from $10 \%$ to $90 \%$ of its peak value and the decrease time of it from $90 \%$ to $10 \%$, respectively. The rise time of $V_{\mathrm{ph}}$ for the BI-2 biointerface at $50 \mathrm{~mW} \mathrm{~cm}{ }^{-2}$ is $58 \mu \mathrm{s}$, which is significantly faster than the $105 \mu$ s rise time of the BI-1 biointerface. Since the photogenerated charges are accumulated at the respective layers via drift during the increase of $V_{\mathrm{oc}}$ and the internal electric field of BI-2 is higher than that of BI-1 (Fig. 2d), the rise time of the negative spike of photocurrent was faster.

When the light is turned off after $10 \mathrm{~ms}$, a complementary behavior of the photocurrent is observed. The fall time of the $\mathrm{BI}-2$ biointerface is recorded as $2.1 \mathrm{~ms}$, which is approximately 3.5-fold faster than the BI-1 biointerface fall time of $7.3 \mathrm{~ms}$. Furthermore, the fall time of BI-2 is $c a$. 36 -fold slower than the rise time because holes and electrons are delocalized in different materials of photoactive layer and ITO/ZnO layers (like in a type-II heterojunction), which require longer time for recombination in comparison with the rise time of the interfacial voltage. The decrease in free charge carrier concentration $\left(n_{\mathrm{c}}\right)$ during recombination implies the increase in effective lifetime $\left(\tau_{\mathrm{n}}\right)$ of the remaining charge carrier recombination $\left(\tau_{\mathrm{n}}=\left(\beta \cdot n_{\mathrm{c}}\right)^{-1}\right.$ where $\beta$, recombination pre-factor), which further slows down the fall time of the photovoltage. ${ }^{34}$ Hence, in addition to the stimuli strength, the biointerface energy levels also influence the switching properties of the capacitive charge-transfer mechanism.

We performed electrochemical impedance spectroscopy (EIS) measurement under light (445 nm, $\left.10 \mathrm{~mW} \mathrm{~cm}^{-2}\right)$ to investigate the charge transfer processes at the biointerfaceelectrolyte interfaces (Fig. $3 \mathrm{a}$ and b). In comparison with BI-1, a lower impedance for BI-2 was observed (Fig. 3a) in the high to mid frequency region, confirming more charge accumulation for the high- $V_{\text {oc }}$ biointerface. The impedance data (Fig. S2, ESI $\dagger$ ), extracted by fitting the experimental data with an equivalent electrical circuit, show that the electrode-electrolyte capacitance of BI-2 $\left(C_{\mathrm{dl}}=2.9 \mu \mathrm{F} \mathrm{cm}{ }^{-2}\right)$ is higher than that of BI-1 $\left(C_{\mathrm{dl}}=2.6 \mu \mathrm{F} \mathrm{cm}^{-2}\right)$, which is another factor leading to higher photocapacitive currents for BI-2. At the same time, the faster response of BI-2 is confirmed by the Bode phase peak frequency at $5.96 \mathrm{kHz}$, which is higher than the peak frequency of BI-1 at $3.72 \mathrm{kHz}$ (Fig. 3b). Hence, BI-2 is a better candidate for neural (a)

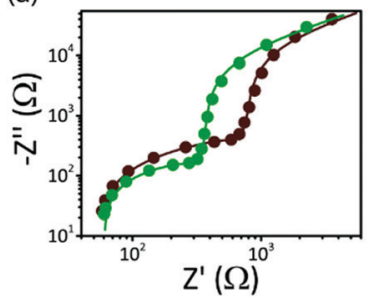

(b)

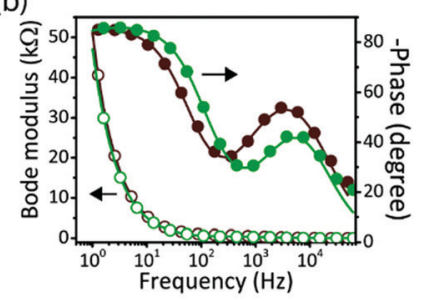

Fig. 3 Electrochemical impedance spectroscopy of biointerfaces at zero dc bias under the illumination of $10 \mathrm{~mW} \mathrm{~cm}^{-2}$ at the wavelength of $445 \mathrm{~nm}$ : (a) Nyquist plot and (b) Bode modulus and Bode phase of the biointerfaces with fitted solid lines. Experimental data are shown by dots and the fitted data are indicated by solid lines. The dark red color represents $\mathrm{BI}-1$ and the dark green color represents $\mathrm{BI}-2$ biointerface.

interface applications because of high current injection levels with fast kinetics.

\section{Light intensity dependent study of high $V_{\text {oc }}$ biointerface}

We initially investigate the light intensity dependent photoresponse of the high- $V_{\mathrm{oc}}$ biointerface (BI-2). In the case of a wired photovoltaic device, the current density can be determined by using eqn (1), where $R_{\mathrm{sh}}$ is the shunt resistance, $R_{\mathrm{S}}$ is the series resistance, $J_{0}$ is the reverse saturation current density, $q$ is the electronic charge, $k_{\mathrm{B}}$ is the Boltzmann constant, $V$ is the output voltage, $T$ is the temperature and $J_{\mathrm{ph}}$ is the photo-current density. ${ }^{34}$ In these photovoltaic devices, direct electron transfer from the device to the external circuitry is responsible for the current. However, in the capacitive photovoltaic biointerfaces since the direct electron transfer is limited due to the low-level faradaic contribution, the biointerfaces operate more closely under the open-circuit conditions. Hence, we can consider that the conduction current is approximately zero and $V \approx V_{\mathrm{oc}}$. Since this case analogically corresponds to a shunt resistance much larger than the series resistance in wired photovoltaic devices, $V_{\text {oc }}$ can be approximated as in eqn (2) showing the relation between the intensity and open-circuit voltage of the biointerface.

$$
J=\left[R_{\mathrm{sh}} /\left(R_{\mathrm{sh}}+R_{\mathrm{s}}\right)\right]\left[J_{0}\left\{\exp \left(q\left(V-J R_{\mathrm{s}}\right) / n k_{\mathrm{B}} T\right)\right\}+\left(V / R_{\mathrm{sh}}\right)\right]-J_{\mathrm{ph}}
$$

$$
V_{\mathrm{oc}} \propto\left(n k_{\mathrm{B}} T / q\right) \ln \left(C\left(I_{\mathrm{ph}} / I_{0}\right)+1\right)
$$

To explore the correlation between the open-circuit voltage of photovoltaic devices and the photovoltage of the biointerface, the photovoltaic device $\mathrm{BI}-2 / \mathrm{MoO}_{x} / \mathrm{Ag}$ was characterized under blue light with similar intensity levels and wavelengths that are used for photocurrent measurement of the biointerface. Fig. 4a compares $V_{\mathrm{ph}}$ and $V_{\mathrm{oc}}$ as a function of light intensity for BI-2. The logarithmic dependence of $V_{\mathrm{oc}}$ and $V_{\mathrm{ph}}$ on incident light intensity was observed, which confirms the direct proportional relationship between $V_{\mathrm{oc}}$ and $V_{\mathrm{ph}}$ and their dependence on light intensity as in eqn (2).

We investigated the photoresponse of the biointerface under different illumination conditions. Fig. 4 b shows the variation of photocurrent for the BI-2 biointerface within the light intensity 
(a)

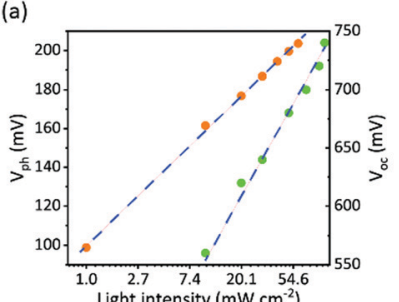

(b)

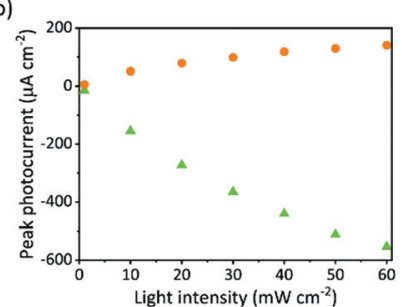

(c)

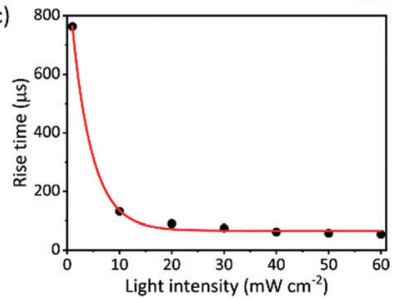

Fig. 4 (a) Photovoltages $\left(V_{\text {ph }}\right)$ and their corresponding open-circuit voltages $\left(V_{o c}\right)$ of high- $V_{o c}$ biointerface under different light intensity levels. Orange dots correspond to the $V_{\mathrm{ph}}$ values, green dots correspond to the $V_{\text {oc }}$ values, and the dotted lines signify the linear fitting. (b) The relationship between light intensity and peak photocurrent for both negative (orange color dots) and positive spikes (green color dots). (c) $V_{\text {ph }}$ rise time for different light intensity levels.

range from $1 \mathrm{~mW} \mathrm{~cm}^{-2}$, which is inside the retinal irradiance levels, to $60 \mathrm{~mW} \mathrm{~cm} \mathrm{~cm}^{-2}$. We observed $14 \mu \mathrm{A} \mathrm{cm}^{-2}$ at a low intensity level of $1 \mathrm{~mW} \mathrm{~cm}^{-2}$, and as the light intensity increases, the peak value of both the negative and positive spikes of the capacitive photocurrent almost linearly increases revealing single photon-induced absorption processes. ${ }^{35,36}$ Moreover, we observed that while the light intensity increases, it leads to a faster switching of capacitive stimuli (Fig. 4c). Under the same range of intensity, the rise time of the BI-2 biointerface induces a larger variation from 762 to $54 \mu \mathrm{s}$. Moreover, since the dependence of the rise time is exponential with the light intensity, this also proves the direct correlation of rise time with open-circuit voltage. Hence, light-intensity defines an unconventional variable of stimulating pulses in electrode-electrolyte junctions.

\section{Viability and electrophysiology of neuron cells}

Before interfacing with cells, we investigated cytotoxic effects of the biointerfaces by assessing the mitochondrial activity of SH-SY5Y cells using MTT assay. ${ }^{9,37}$ Cells grown on BI-2 biointerfaces exhibited comparable cell viability with respect to BI-1 and ITO controls suggesting that the biointerfaces do not exhibit any toxic effect on the cells (Fig. S3a, ESI $\dagger$ ). Furthermore, the morphology of the cells grown on the photoelectrodes was assessed using immunolabelling and fluorescence microscopy. Beta-III-tubulin primary antibody was used as a neuronal marker; and DAPI staining was performed for nucleus visualization. The results suggested no morphological difference between cells grown on the control substrate and cells grown on the photoelectrodes (Fig. S3b, ESI $\dagger$ ). Moreover, a detailed stability study of the organic semiconductor materials with thiophene molecules as the building block in biological medium is discussed by Han et al., in which the (a)

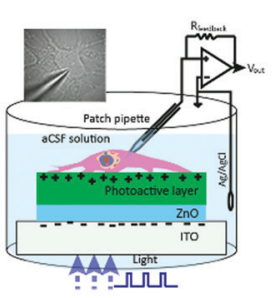

(b)

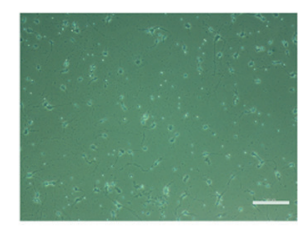

(d)

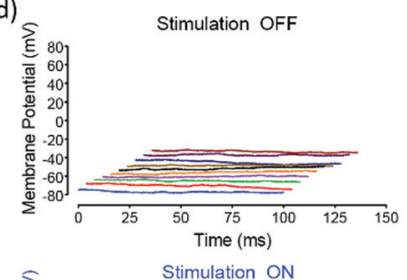

(c)

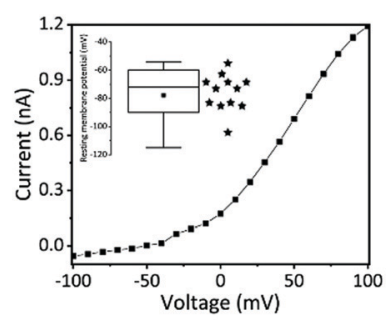

(e)

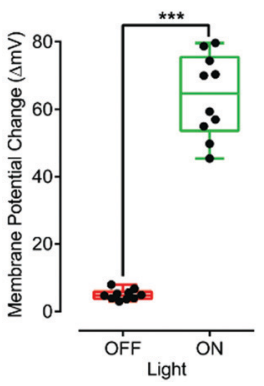

Fig. 5 (a) Schematic of the whole-cell patch clamp recording configuration of the biointerface in free-standing mode. The cells are grown on the biointerface, and adhere to the biointerface. A LED at far field is used to illuminate and activate the biointerface. Primary hippocampal neurons were incubated for 3 days on the photoelectrode and afterward, they were patched (as shown in the inset) and recorded under the whole-cell configuration. (b) A phase-contrast light microscope image of the primary hippocampal neurons on the photoelectrode. Scale bar is $200 \mu \mathrm{m}$. (c) Typical IV-characteristics of the primary hippocampal neurons recorded in the voltage-clamp configuration under dark conditions. Inset shows the resting membrane potential distribution for $N=12$ cells. (d) 3D plots for light-evoked membrane potential modulation in the current-clamp configuration subjected to $1 / 0.1 \mathrm{~ms}$ light stimulation (burst waveform). The top panel shows the light-triggered neural activity of cells cultured on the Petri dish. The bottom panel shows action potential generation after the light illumination $(N=10)$. Each color shows the separate response of distinct neurons on the biointerface. (e) Box plot graphics of light stimulation related membrane potential change $(\mathrm{mV})$ in primary hippocampal neurons. The neurons displaying a significant (two-sided Student's $t$-test) light induced membrane potential increase compared to the control $\left({ }^{* \star *} p<0.0001, N=10\right)$.

time-dependent cyclic stability by measuring the peak value of the transient photocurrent is presented. ${ }^{38}$ The recorded peak photocurrent density only decreased by $9.2 \%$ after 60 days, relative to the first day, which corresponds to a device half-time of $\sim 1.8$ years in an aqueous environment.

To investigate the membrane potential variation and generation of action potential, we used primary hippocampal neurons extracted from decapitated E15-E17 Wistar Albino rats. We performed single-cell patch clamping in whole-cell configuration in the current clamp mode $(I=0)$ (Fig. 5a). The image (in the inset of Fig. 5a) shows a patched primary hippocampal neuron during the electrophysiological recording. The primary cells are grown on the reference substrate that 
consists of the Petri dish and the BI-2 biointerface that consists of photoactive surface. Fig. 5b shows the phase-contrast light microscope image of fully grown primary hippocampal cells on the biointerface, which shows that the neurons are significantly separated without synaptic connections. Fig. 5c shows typical IV-characteristics of primary hippocampal cells from the BI-2 biointerface, which were measured with a voltage clamp under dark conditions. The cells have quasi-linear response around the resting potential. The average resting potential of the cells $(N=12)$ was observed as $-78 \mathrm{mV}$.

Similar to the photocurrent experiments, the electrophysiology is performed in the wireless and free-standing mode, and light pulses with an intensity level of $20 \mathrm{~mW} \mathrm{~cm}^{-2}$ were used for the photostimulation experiments. ${ }^{9,39}$ Before performing the electrophysiology experiment with BI-2 biointerface, the reference substrate (primary hippocampal cells grown on Petri dish) is subjected to light stimulation as a control experiment. When the light is illuminated on the cells $(N=3)$ with varying stimulation pattern, there was no neural activity as shown in Fig. S4 (ESI $\dagger$ ). This confirms that the photostimulation of neurons alone does not show any spontaneous activity.

Furthermore, the photostimulation experiment was done on the BI-2. The top panel of Fig. 5d shows no neural photostimulation when light illumination was applied. When the light was illuminated on the biointerface, the photogenerated excitons are separated at the donor-acceptor interfaces and according to the energy diagram electrons were guided towards the ITO electrode because of the electron transport layer $(\mathrm{ZnO})$, and holes were transferred toward the respective higher hole energy molecule of PTB7-Th for the BI-2 biointerface (Fig. 2d). The accumulation of holes attracted the negative ions inside the electrolyte at the semiconductor-electrolyte interface. This generated a displacement current with a direction from the semiconductor towards the electrolyte. As per our assumption, it should lead to hyperpolarization of the attached membrane and depolarization of the free membrane. Since the longer light pulse (10 ms on-time and $10 \mathrm{~ms}$ off-time) with square waveform has shown marginal neuromodulation at $20 \mathrm{~mW} \mathrm{~cm}^{-2}$, the action potential was generated when a burst waveform (1 ms on-time and $0.1 \mathrm{~ms}$ off-time) was applied (Fig. $5 \mathrm{~d}$ bottom panel), which has been previously proposed and demonstrated by Fromherz and co-workers by using electrical stimulation. ${ }^{40}$ This is the first demonstration of using burst light pulses with a high- $V_{\text {oc }}$ photovoltaic biointerface for capacitive photostimulation that results in action potential generation at low light intensity levels. Since the biointerface has a faster switching response ( $c a .50 \mu$ s light pulses) and the capacitive current relies on the time-dependent variation of the photovoltage change, it is one of the reasons of photostimulation at low light intensities. The stimulation is mainly triggered due to the repetitive activation of sodium channels and a summation of small inward current pulses. ${ }^{40,41}$ In addition to the 3D plots for light-evoked membrane potential modulation, we provided an isolated single plot during the photostimulation as shown in Fig. S5 (ESI $\dagger$ ).

Fig. 5e shows the statistical analysis that was performed using two-sided Student's $t$-test for voltage patch-clamp results on primary hippocampal neurons to determine the degree of significance (Prism 7; GraphPad, San Diego, CA, USA). Results were considered statistically significant when the $p$-value was less than 0.01 . The primary hippocampal neurons displayed a significant (average $\sim 65 \mathrm{mV}$, range $\sim 45-80 \mathrm{mV}$ ) light-induced membrane potential increase compared to the control experiment.

\section{Discussion}

Interestingly, the OFF current behaviour is similar for the two systems (Fig. 2c). This is possibly due to the fact that the comparable absorption levels lead to generation of a similar number of photogenerated charge carrier and similar levels of charge carrier recombination occur in both systems. The effective lifetime $\left(\tau_{\mathrm{n}}\right)$ of the remaining charge carrier recombination is given by $\tau_{\mathrm{n}}=\left(\beta \cdot n_{\mathrm{c}}\right)^{-1}$ where $\beta$ is the recombination pre-factor, ${ }^{34}$ and the magnitudes of the effective lifetime are comparable for both BI-1 (P3HT:PCBM) and BI-2 (PTB7Th:PC71BM) photoactive materials because the recombination pre-factor and charge carrier density of states for both bulk heterojunctions are on the same order of magnitude as $10^{-17} \mathrm{~m}^{3} \mathrm{~s}^{-1}$ and $10^{17} \mathrm{~cm}^{-3}$, respectively. ${ }^{19,21,42}$ Hence, when the light is turned OFF, the photogenerated electrons and hole concentrations that are localized in the acceptor and donor molecules similarly decrease both in the BI-1 and BI-2 interfaces.

Other than organic semiconductors, a variety of inorganic semiconductors, especially multiscale silicon structures, have been investigated for neuron photostimulation. Bulk inorganic semiconductors are rigid and they have a high elastic modulus of about $100 \mathrm{GPa}$, whereas the neural and other tissues exhibit elastic moduli in the kilo to megapascal range. ${ }^{43,44}$ Hence, bulk silicon-based implanted probes have elastic mismatch with neural tissues, which can damage the surrounding neurons around the implant resulting in a reduction in the utility of the neuron interface over time. ${ }^{45-47}$ Alternatively, a variety of wirefree silicon configurations can be used to seamlessly interface with biological systems for photostimulation. Si nanowires allows for intracellular uptake and interfacing with mammalian cells. ${ }^{48}$ Moreover, planar Si structures can be used to build extracellular interfaces with single cells or small tissues. Furthermore, flexible devices made of Si membrane on holey PDMS membrane can lead to conformal contacts with soft tissues (e.g., mouse brain cortex). ${ }^{39}$ Hence, beside organic semiconductor systems, a wide variety of inorganic or inorganic-organic hybrid systems can be engineered for modulation of living systems with light. However, organic semiconductor systems could be preferred over the inorganic counterparts (having mechanical flexibility) because of significant elastic mismatch, a material property, with neural tissues.

\section{Conclusions}

To avoid any damage on the electrode due to corrosion or on the cell due to faradaic reactions, the stimuli must be as weak 
as possible. Hence, optical excitation at low light intensity levels with burst waveforms allows for a safe and efficient neuromodulation scheme. During optical excitation, photothermal effects may also contribute to the stimulation of neurons. At high intensity levels, it may induce phase transition in the phospholipid ordering, change in membrane conductance and rearrangement of the ions. ${ }^{49,50}$ To explore the thermal effects, we calculated the heat generated at the biointerface surface due to $20 \mathrm{~mW} \mathrm{~cm}^{-2}$ optical excitation of blue light. We found that the increase in temperature is merely $0.001{ }^{\circ} \mathrm{C}$ (Appendix-1, ESI $\dagger$ ), which is insufficient for such a photothermal stimulation of the cells. Moreover, the level of intensities that we use in this study is comparatively much lower than the reports studying the thermal activation of neurons. ${ }^{51}$

In this study, we introduced a new photoactive bulkheterojunction material system, PTB7-Th:PC71BM, for photostimulation of cells and we incorporated it into a capacitive charge-injecting biointerface design. By using this biointerface, we investigated the effect of the open-circuit voltage on the photoresponse of the bulk-heterojunction, and we observed that high- $V_{\text {oc }}$ can simultaneously lead to strong and fast capacitive currents at the electrode-cell interfaces. In addition, light can also control the switching speed of capacitive currents that are caused by the variation of open-circuit voltage. Hence, the open-circuit voltage is an important design parameter for fast and effective switching of the neurons that can be controlled by light and electronic energy levels of the heterojunctions. Also, some of the visual attributes can only be perceived at the threshold for the specific light pulses (in sub- $\mu$ s region) and train frequency. ${ }^{18,52,53}$ Hence, the high- $V_{\text {oc }}$ biointerface can satisfy these crucial aspects as well. Moreover, the wide absorption ranges from visible to near IR of the photoactive material system can be utilized for deep-tissue photostimulation such as the brain and heart. Therefore, bulk heterojunction composite based biointerfaces have high potential for safe, rapid, and controlled neural photostimulation of cells.

\section{Conflicts of interest}

There are no conflicts to declare.

\section{Acknowledgements}

This project has received funding from the European Research Council (ERC) under the European Union's Horizon 2020 Research and Innovation Programme (grant agreement no. 639846). S. N. acknowledges the support by the Turkish Academy of Sciences (TÜBA-GEBIP; The Young Scientist Award Program) and the Science Academy of Turkey (BAGEP; The Young Scientist Award Program). The authors thank Dr M. Barış Yağcı for scanning electron microscopy measurements, and Koç University Surface Science and Technology Center (KUYTAM) for providing the SEM measurement infrastructure. The authors thank Mr Mertcan Han for helping in the solar cell measurement. The authors gratefully acknowledge the use of the services and facilities of the Koç University Research Center for Translational Medicine (KUTTAM), funded by the Republic of Turkey Ministry of Development. The content is solely the responsibility of the authors and does not necessarily represent the official views of the Ministry of Development. All experimental procedures have been approved by the Institutional Animal Care and Use Committees of Koç University (Approval No: 2019.HADYEK.023) according to Directive 2010/63/EU of the European Parliament and of the Council on the Protection of Animals Used for Scientific Purposes.

\section{Notes and references}

1 G. A. Goetz and D. V. Palanker, Rep. Prog. Phys., 2016, 79, 096701.

2 G. Lanzani, Nat. Mater., 2014, 13, 775.

3 P. Fattahi, G. Yang, G. Kim and M. R. Abidian, Adv. Mater., 2014, 26, 1846-1885.

4 Y. Hanein and L. Bareket-Keren, Front. Neural Circuits, 2013, 6, 122.

5 M. Choi, J. W. Choi, S. Kim, S. Nizamoglu, S. K. Hahn and S. H. Yun, Nat. Photonics, 2013, 7, 987.

6 K. Mathieson, J. Loudin, G. Goetz, P. Huie, L. Wang, T. I. Kamins, L. Galambos, R. Smith, J. S. Harris, A. Sher and D. Palanker, Nat. Photonics, 2012, 6, 391-397.

7 R. M. Owens and G. G. Malliaras, MRS Bull., 2010, 35, 449-456.

8 J. F. Maya-Vetencourt, D. Ghezzi, M. R. Antognazza, E. Colombo, M. Mete, P. Feyen, A. Desii, A. Buschiazzo, M. Di Paolo, S. Di Marco, F. Ticconi, L. Emionite, D. Shmal, C. Marini, I. Donelli, G. Freddi, R. Maccarone, S. Bisti, G. Sambuceti, G. Pertile, G. Lanzani and F. Benfenati, Nat. Mater., 2017, 16, 681.

9 S. B. Srivastava, R. Melikov, M. M. Aria, U. M. Dikbas, I. H. Kavakli and S. Nizamoglu, Phys. Rev. Appl., 2019, 11, 044012.

10 G. Simone, D. Di Carlo Rasi, X. de Vries, G. H. L. Heintges, S. C. J. Meskers, R. A. J. Janssen and G. H. Gelinck, Adv. Mater., 2018, 30, 1804678.

11 G. Yu, J. Gao, J. C. Hummelen, F. Wudl and A. J. Heeger, Science, 1995, 270, 1789.

12 V. Gautam, D. Rand, Y. Hanein and K. S. Narayan, Adv. Mater., 2014, 26, 1751-1756.

13 D. Ghezzi, M. R. Antognazza, R. Maccarone, S. Bellani, E. Lanzarini, N. Martino, M. Mete, G. Pertile, S. Bisti, G. Lanzani and F. Benfenati, Nat. Photonics, 2013, 7, 400.

14 D. Ghezzi, M. R. Antognazza, M. Dal Maschio, E. Lanzarini, F. Benfenati and G. Lanzani, Nat. Commun., 2011, 2, 166.

15 V. Gautam, M. Bag and K. S. Narayan, J. Am. Chem. Soc., 2011, 133, 17942-17949.

16 M. R. Antognazza, D. Ghezzi, D. Musitelli, M. Garbugli and G. Lanzani, Appl. Phys. Lett., 2009, 94, 243501.

17 Y. Yan, F. Cai, L. Yang, J. Li, Y. Zhang, F. Qin, C. Xiong, Y. Zhou, D. G. Lidzey and T. Wang, Adv. Mater., 2017, 29, 1604044 . 
18 S. F. Cogan, Annu. Rev. Biomed. Eng., 2008, 10, 275-309.

19 S. B. Srivastava, S. K. Srivastava and S. P. Singh, J. Phys. Chem. C, 2017, 121, 17104-17111.

20 S. B. Srivastava, P. Sonar and S. P. Singh, AIP Adv., 2015, 5, 077177.

21 G. Garcia-Belmonte, A. Munar, E. M. Barea, J. Bisquert, I. Ugarte and R. Pacios, Org. Electron., 2008, 9, 847-851.

22 D. Rand, M. Jakešová, G. Lubin, I. Vẻbraitè, M. David-Pur, V. Đerek, T. Cramer, N. S. Sariciftci, Y. Hanein and E. D. Głowacki, Adv. Mater., 2018, 30, 1707292.

23 M. L. Seibenhener and M. W. Wooten, J. Visualized Exp., 2012, 65, e3634, DOI: 10.3791/3634.

24 A. M. Kleman, J. Y. Yuan, S. Aja, G. V. Ronnett and L. E. Landree, J. Neurosci. Methods, 2008, 167, 292-301.

25 V. Sample, S. Ramamurthy, K. Gorshkov, G. V. Ronnett and J. Zhang, Mol. Biol. Cell, 2015, 26, 1935-1946.

26 M. C. Scharber, D. Mühlbacher, M. Koppe, P. Denk, C. Waldauf, A. J. Heeger and C. J. Brabec, Adv. Mater., 2006, 18, 789-794.

27 J.-L. Brédas, D. Beljonne, V. Coropceanu and J. Cornil, Chem. Rev., 2004, 104, 4971-5004.

28 N. S. Sariciftci, L. Smilowitz, A. J. Heeger and F. Wudl, Science, 1992, 258, 1474.

29 S.-H. Liao, H.-J. Jhuo, Y.-S. Cheng and S.-A. Chen, Adv. Mater., 2013, 25, 4766-4771.

30 D. Chi, S. Qu, Z. Wang and J. Wang, J. Mater. Chem. C, 2014, 2, 4383-4387.

31 K. Yuan, L. Chen and Y. Chen, J. Mater. Chem. C, 2014, 2, 3835-3845.

32 A. Rizzo, A. Cester, N. Wrachien, N. Lago, L. Torto, M. Barbato, J. Favaro, S. A. Gevorgyan, M. Corazza and F. C. Krebs, Sol. Energy Mater. Sol. Cells, 2016, 157, 337-345.

33 P. R. Berger and M. Kim, J. Renewable Sustainable Energy, 2018, 10, 013508.

34 N. K. Elumalai and A. Uddin, Energy Environ. Sci., 2016, 9, 391-410.

35 A. K. K. Kyaw, D. H. Wang, V. Gupta, W. L. Leong, L. Ke, G. C. Bazan and A. J. Heeger, ACS Nano, 2013, 7, 4569-4577.

36 Z. Li, F. Gao, N. C. Greenham and C. R. McNeill, Adv. Funct. Mater., 2011, 21, 1419-1431.

37 M. M. Aria, S. B. Srivastava, E. Sekerdag, U. M. Dikbas, S. Sadeghi, S. R. Pering, P. J. Cameron, Y. Gursoy-Ozdemir, I. H. Kavakli and S. Nizamoglu, Adv. Mater. Interfaces, 2019, 6, 1900758.
38 M. Han, S. B. Srivastava, E. Yıldız, R. Melikov, S. Surme, I. B. Dogru-Yuksel, I. H. Kavakli, A. Sahin and S. Nizamoğlu, ACS Appl. Mater. Interfaces, 2020, 12(38), 42997-43008, DOI: 10.1021/acsami.0c11581.

39 Y. Jiang, X. Li, B. Liu, J. Yi, Y. Fang, F. Shi, X. Gao, E. Sudzilovsky, R. Parameswaran, K. Koehler, V. Nair, J. Yue, K. Guo, Y. Fang, H.-M. Tsai, G. Freyermuth, R. C. S. Wong, C.-M. Kao, C.-T. Chen, A. W. Nicholls, X. Wu, G. M. G. Shepherd and B. Tian, Nat. Biomed. Eng., 2018, 2, 508-521.

40 I. Schoen and P. Fromherz, J. Neurophysiol., 2008, 100, 346-357.

41 S. B. Srivastava, R. Melikov, E. Yildiz, M. Han, A. Sahin and S. Nizamoglu, Biomed. Opt. Express, 2020, 11, 5237-5248.

42 D. Bartesaghi, I. D. C. Pérez, J. Kniepert, S. Roland, M. Turbiez, D. Neher and L. J. A. Koster, Nat. Commun., 2015, 6, 7083.

43 B. Tian, S. Xu, J. A. Rogers, S. Cestellos-Blanco, P. Yang, J. L. Carvalho-de-Souza, F. Bezanilla, J. Liu, Z. Bao, M. Hjort, Y. Cao, N. Melosh, G. Lanzani, F. Benfenati, G. Galli, F. Gygi, R. Kautz, A. A. Gorodetsky, S. S. Kim, T. K. Lu, P. Anikeeva, M. Cifra, O. Krivosudský, D. Havelka and Y. Jiang, Phys. Biol., 2018, 15, 031002.

44 T. Someya, Z. Bao and G. G. Malliaras, Nature, 2016, 540, 379-385.

45 V. S. Polikov, P. A. Tresco and W. M. Reichert, J. Neurosci. Methods, 2005, 148, 1-18.

46 H. Lee, R. V. Bellamkonda, W. Sun and M. E. Levenston, J. Neural Eng., 2005, 2, 81-89.

47 D. H. Szarowski, M. D. Andersen, S. Retterer, A. J. Spence, M. Isaacson, H. G. Craighead, J. N. Turner and W. Shain, Brain Res., 2003, 983, 23-35.

48 M. Y. Rotenberg, B. Elbaz, V. Nair, E. N. Schaumann, N. Yamamoto, N. Sarma, L. Matino, F. Santoro and B. Tian, Nano Lett., 2020, 20, 1226-1232.

49 M. Plaksin, E. Shapira, E. Kimmel and S. Shoham, Phys. Rev. $X, 2018,8,011043$.

50 A. Varghese, E. M. TenBroek, J. Coles and D. C. Sigg, Prog. Biophys. Mol. Biol., 2006, 90, 26-37.

51 N. Martino, P. Feyen, M. Porro, C. Bossio, E. Zucchetti, D. Ghezzi, F. Benfenati, G. Lanzani and M. R. Antognazza, Sci. Rep., 2015, 5, 8911.

52 C. Veraart, C. Raftopoulos, J. T. Mortimer, J. Delbeke, D. Pins, G. Michaux, A. Vanlierde, S. Parrini and M.-C. Wanet-Defalque, Brain Res., 1998, 813, 181-186.

53 F. T. Hambrecht, Bailliere. Clin. Neurol., 1995, 4, 147-165. 